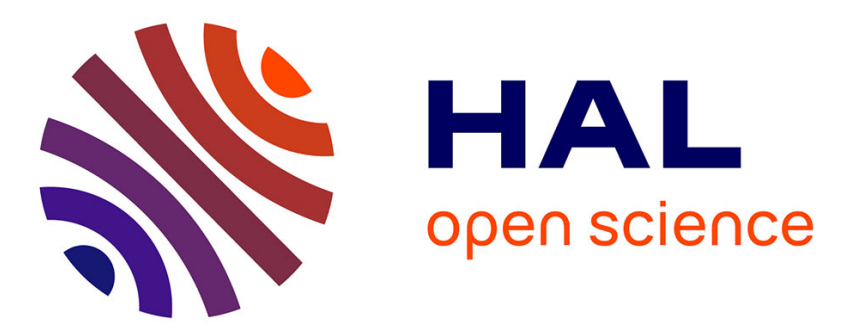

\title{
A line integral approach for the computation of the potential harmonic coefficients of a constant density polyhedron
}

Olivier Jamet, Dimitrios Tsoulis

\section{- To cite this version:}

Olivier Jamet, Dimitrios Tsoulis. A line integral approach for the computation of the potential harmonic coefficients of a constant density polyhedron. Journal of Geodesy, 2020, 94 (3), pp.30. 10.1007/s00190-020-01358-8 . hal-02612186

\section{HAL Id: hal-02612186 \\ https://hal.science/hal-02612186}

Submitted on 19 May 2020

HAL is a multi-disciplinary open access archive for the deposit and dissemination of scientific research documents, whether they are published or not. The documents may come from teaching and research institutions in France or abroad, or from public or private research centers.
L'archive ouverte pluridisciplinaire HAL, est destinée au dépôt et à la diffusion de documents scientifiques de niveau recherche, publiés ou non, émanant des établissements d'enseignement et de recherche français ou étrangers, des laboratoires publics ou privés. 


\title{
A line integral approach for the computation of the potential harmonic coefficients of a constant density polyhedron
}

\author{
Olivier Jamet ${ }^{1,2}$ and Dimitrios Tsoulis ${ }^{3}$ \\ ${ }^{1}$ Université de Paris, Institut Physique du Globe de Paris, CNRS, \\ IGN, F-75238 Paris, France \\ ${ }^{2}$ ENSG-Géomatique, IGN, F-77455 Marne-la-Vallée, France \\ ${ }^{3}$ Department of Geodesy and Surveying, School of Rural and \\ Surveying Engineering, Aristotle University of Thessaloniki
}

July 2019

\begin{abstract}
A novel approach for the computation of the spherical harmonic coefficients of the gravity field of a constant density polyhedron is presented. Based on the expression of the solid harmonics involved in the integrals in term of a gradient of the same function of higher degree, and on the homogeneity and harmonicity of theses functions, the approach leads, after subsequent application of the divergence theorem in 3D and the Stokes theorem on the plane, to explicit line integrals defined along each polyhedral face, which are then summed for all faces of the polyhedral source. In contrast to previous approaches that involved recurrent relations for the integrals of the same functions, the proposed algorithm concludes to a numerical computation of line integrals linked directly to the coefficients. The performed numerical implementation revealed the stability of the proposed algorithm up to degree 360 for a prismatic test source.
\end{abstract}

\section{Introduction}

The problem of computing the spherical harmonic coefficients of the gravity field of a constant density polyhedral source has been considered by many contributions in the past.

First computations, based on the series expansion of the surface of the body itself were implemented as early as 1989 (Chao and Rubincam, 1989; Martinec et al., 1989; Balmino, 1994). Their approach is efficient by poorly adapted to isolated bodies away from from the origin of the reference frame, such as geological layers. 
The problem of the numerical computation of the coefficient through explicit integration has also be tackled very early. The first solution was proposed by Werner (1997). His stategy was based on the decomposition of the body into tetrahedra, and of the integrands into homogeneous monomials that can be integrated analytically. Recent works proposed also a generalized algorithm for integrating homogeneous polynomials inside a space of any dimension (De Loera et al., 2013).

However, the complexity of this family of approaches, which is in the order of $n^{4}$ to compute all the coefficients at degree and order $n$, do not allow very high degree expansions.

Another family of approaches was initiated by Jamet and Thomas (2004) who proposed a linear reccurent scheme, of complexity $n^{2}$ for the computation of all the coefficients at degree and order $n$. Their algorithms was also based on the division of the polyhedron into tetrahedra. In each tetrahedron, the volume integrals were transformed into surface integrals thanks to the divergence theorem, and the surface intergals at degree $n$ were expressed as function of the surface integrals at degree $n-1$ and of line integrals. This method was implemented by Tsoulis et al. (2009), who proved that it was unstable, but that it allowed the computation of the coefficients up to degree 60 for a test asteroid model.

Recently, the work of Tsoulis et al. (2009) was further developed by Chen et al. (2019), who stabilized the recurrent computation by choosing a specific frame for each face and extending the approach to linearly varying densities.

The aforementioned works take benefit from the fact that the involved integrands are homogeneous functions. The application of the divergence theorem to theses functions in 3 dimensions is indeed a particular case of a genegal property of homogeneous functions, which allows their volume integration through the integration over the volume's surface, irrespective of the dimension of the considered space (Lasserre, 1999).

In this work, we show that, in three dimensional spaces, the specific properties of the integrands allow to transform the volume integrals, that express the polyhedral potential harmonic coefficients, into line integrals without the need of a recurrence relation for their numerical evaluation. Theses findings provide the basis for a new computation approach.

After presenting the adopted notations (section 2), we present a comprehensive ouline of the proposed method (section 3). Section 4 gives the analytical expressions of the involved integrals. These expressions are then tested through a numerical experiment on a simple body (section 5).

\section{Notations}

In the present work, we consider the general polyhedral source illustrated in figure 1.

The problem we are adressing was exposed previously by Tsoulis et al. (2009). The spherical harmonic decomposition of the gravitationnal potential $V(r, \theta, \lambda)$ 


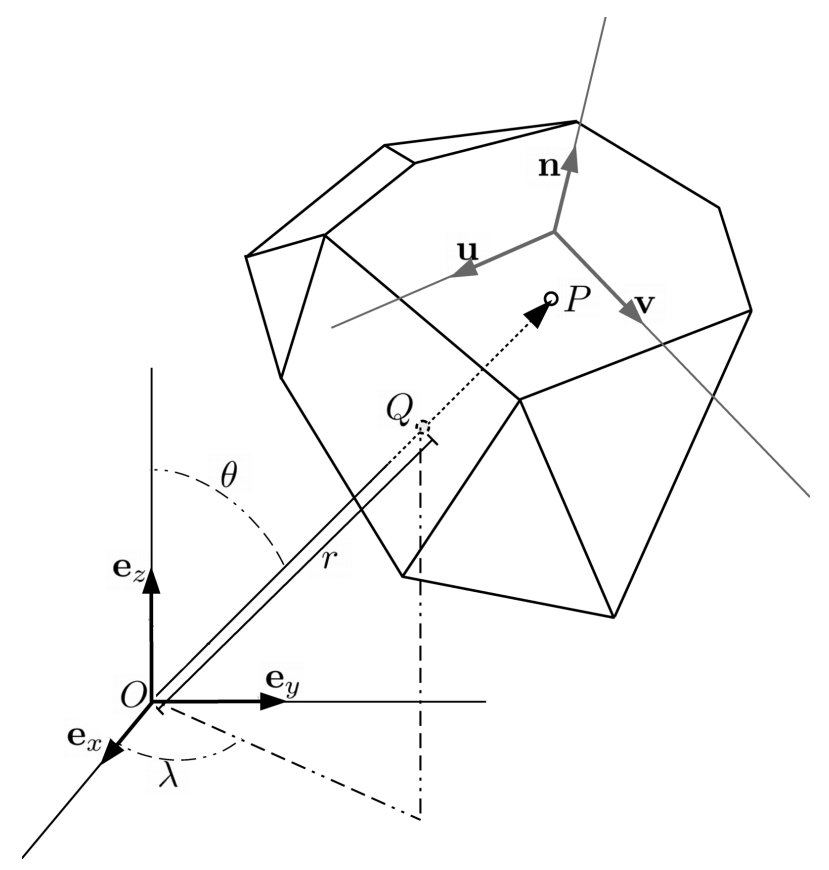

Figure 1: A polyhedron and its associated coordinate frames

of a polyhedral body $\mathcal{U}$ is defined by a set of coefficients $\left\{\left(C_{n, m}, S_{n, m}\right)\right\}_{n, m}$ as

$$
\begin{aligned}
& V(r, \theta, \lambda)=\left\{1+\sum_{n=1}^{+\infty}\left(\frac{a}{r}\right)^{n}\right. \\
&\left.\sum_{m=0}^{n} P_{n, m}(\cos \theta)\left[C_{n, m} \cos (m \lambda)+S_{n, m} \sin (m \lambda)\right]\right\}
\end{aligned}
$$

where $G$ is the gravitational constant, $M$ the total mass of the body and $a$ the radius of a sphere centered at the origin which circumscribes the extended body. $(\mathrm{r}, \vartheta, \lambda)$ are the spherical coordinates of the field point (radius, colati- tude and longitude, respectively, with respect to the coordinate origin $O$, according to figure 1) and $P_{n, m}$ are the Legendre polynomials (for $\mathrm{m}=0$ ) or associated Legendre functions (for $\mathrm{m}>0$ ) as defined by Heiskanen and Moritz (1967).

As stated before by Tsoulis et al. (2009), the coefficients $\left\{\left(C_{n, m}, S_{n, m}\right)\right\}_{n, m}$ can be expressed as

$$
\left[\begin{array}{c}
C_{n, m} \\
S_{n, m}
\end{array}\right]=\frac{2-\delta_{0, m}}{M a^{n}} \frac{(n-m) !}{(n+m) !} \iiint_{Q \in \mathcal{U}}\left[\begin{array}{c}
h_{n, m}^{c}(Q) \\
h_{n, m}^{s}(Q)
\end{array}\right] d u(Q)
$$

where $d u(Q)$ is the volume element of the total volume $\mathcal{U}$ at point $Q$, and where the function $h_{n, m}^{c}$ and $h_{n, m}^{s}$ are defined as

$$
h_{n, m}^{c}(r, \theta, \lambda)=r^{n} P_{n, m}(\cos \theta) \cos (m \lambda)
$$




$$
h_{n, m}^{s}(r, \theta, \lambda)=r^{n} P_{n, m}(\cos \theta) \sin (m \lambda)
$$

Let us note $h_{n, m}$ any of the $h_{n, m}^{c}$ or $h_{n, m}^{s}$ functions. Tsoulis et al. (2009) showed that the integral of $h_{n, m}$ over the volume $\mathcal{U}$ could be expressed as a surface integral through the relation

$$
\begin{aligned}
\iiint_{\mathcal{U}} h_{n, m}(Q) d u(Q) & =\frac{1}{n+3} \oiint_{\partial \mathcal{U}} h_{n, m}(P) \mathbf{O P} \cdot \mathbf{d} \mathbf{S}(P) \\
& =\frac{1}{n+3} \sum_{\mathcal{F} \in \partial \mathcal{U}} \iint_{\mathcal{F}} h_{n, m} \mathbf{r} \cdot \mathbf{n} d S \\
& =\frac{1}{n+3} \sum_{\mathcal{F} \in \partial \mathcal{U}} d_{\mathcal{F}} \iint_{\mathcal{F}} h_{n, m} d S
\end{aligned}
$$

where $P$ is a point of the surface $\partial \mathcal{U}, \mathbf{O P} \equiv \mathbf{r}$ is the vector from the origin $O$ to the point $P, \mathbf{n}$ is the unitary vector normal to the surface $\partial \mathcal{U}, d S$ is the surface element at point $P$ (see figure 1$), \mathcal{F}$ is a given planar face of the polyhedron, and $d_{\mathcal{F}}$ is the algebric distance from the origin $O$ to the plane bearing the planar face $\mathcal{F}$ : for any point $P$ on the face $\mathcal{F}, d_{\mathcal{F}}=\mathbf{O P} \cdot \mathbf{n}_{\mathcal{F}}$, with $\mathbf{n}_{\mathcal{F}}$, the oriented unit normal vector to the face.

The problem of computing the coefficients is thus equivalent to the problem of computing on any plolyhedron face $\mathcal{F}$ the integral $I_{n, m}$ defined as

$$
I_{n, m}=\iint_{\mathcal{F}} h_{n, m} d S
$$

In the following, we denote vectors with a boldface typesetting. The natural basis of the considered $3 D$ space, associated to the cartesian coordinates $(x, y, z)$, will be noted $\left(\mathbf{e}_{x}, \mathbf{e}_{y}, \mathbf{e}_{z}\right)$. For a given face $\mathcal{F}$ of the boundary of the polyhedron, the normal vector will be written $\mathbf{n}$, or $\mathbf{n}_{\mathcal{F}}$ when several faces are involved. The edges of the face $\mathcal{F}$ will be noted $\{\mathcal{E}\}_{\mathcal{E} \in \partial \mathcal{F}}$. For each edge $\mathcal{E}$, the leading unit oriented vector will be written $\mathbf{u}_{\mathcal{E}}$.

We will use the $\nabla$ operator to denote the gradient of a scalar field $f(\nabla f)$ or the divergence of a vector field $\mathbf{F}(\nabla \cdot \mathbf{F})$. We will use the subscript 2 (for instance $\left.\nabla_{2} f\right)$ to indicate when the $\nabla$ operator applies in a 2 dimension space.

\section{Outline of our approach}

The purpose of this section is to give a comprehensive explanation of the proposed method. A mathematical formulation is briefly presented in section 4.

The proposed approach makes use of the following relation that allows to express $h_{n, m}$ as a derivative oh $h_{n+1, m}$ (see demonstration in the appendix, section A.1).

$$
\nabla h_{n+1, m} \cdot \mathbf{e}_{z}=\frac{\partial h_{n+1, m}}{\partial z}=(n+m+1) h_{n, m}
$$




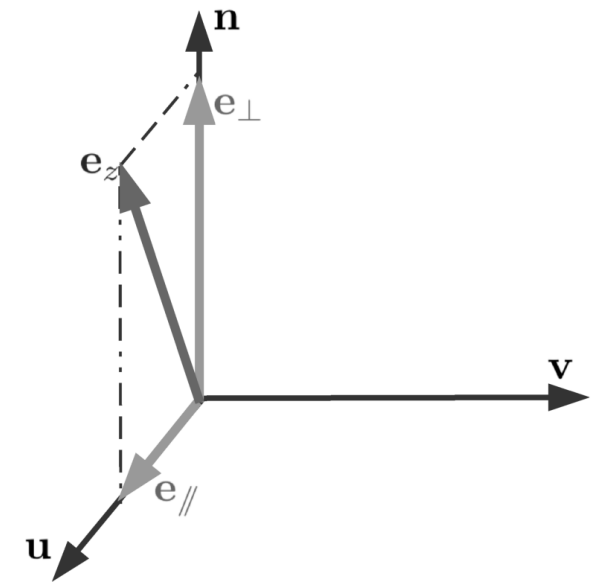

Figure 2: Decomposition of the unit vector $\mathbf{e}_{z}$ into two parts: $\mathbf{e}_{\perp}$, perpendicular to the face, and $\mathbf{e}_{/ /}$, parallel to the face.

Expressed that way, the integral is first decomposed into two parts, one using the derivative along the normal to the face, and the second one using the derivative along a vector parallel to the face. These two parts are shown to be easily expressed as sums of line integrals.

\subsection{Decomposition}

$\mathcal{F}$ being a planar face of normal unitary vector $\mathbf{n}$, the vector $\mathbf{e}_{z}$ can be decomposed on the whole integration domain as

$$
\mathbf{e}_{z}=\mathbf{e}_{\perp}+\mathbf{e}_{/ /}
$$

where $\mathbf{e}_{\perp}$ is othogonal to the plane bearing the face $\mathcal{F}$, and $\mathbf{e}_{/ /}$is parallel to this plane (figure 2). Theses two components of $\mathbf{e}_{z}$ are thus defined by relation 8 and by

$$
\begin{array}{lll}
\mathbf{e}_{\perp} & / / & \mathbf{n} \\
\mathbf{e}_{/} & \perp & \mathbf{n}
\end{array}
$$

We thus decompose the surface integration into two parts $I_{n \perp}$ and $I_{n / /}$ defined as

$$
\begin{aligned}
I_{n} & =\frac{1}{n+m+1}\left(I_{n \perp}+I_{n / /}\right) \\
I_{n \perp} & =\iint_{\mathcal{F}} \nabla h_{n+1, m} \cdot \mathbf{e}_{\perp} d S \\
I_{n / /} & =\iint_{\mathcal{F}} \nabla h_{n+1, m} \cdot \mathbf{e}_{/ /} d S
\end{aligned}
$$




\subsection{Parallel part}

The reduction of the dimension of the integral for the parallel part is straightforward. Let $(\mathbf{u}, \mathbf{v})$ be an orthonormal frame of the face $\mathcal{F}$, associated to the cartesian coordinates $(u, v)$, chosen so as

$$
\mathbf{e}_{/ /}=\| \mathbf{e}_{/ / \| \mathbf{u}}
$$

The $I_{n / /}$ integral can be written as

$$
I_{n / /}=\left\|\mathbf{e}_{/ /}\right\| \iint_{\mathcal{F}} \frac{\partial h_{n+1, m}}{\partial u} d u d v
$$

Noting that in the $2 D$ space defined by the plane of the face $\mathcal{F}$, the derivative $\frac{\partial h_{n+1, m}}{\partial u}$ can be expressed as a divergence

$$
\frac{\partial h_{n+1, m}}{\partial u}=\nabla_{2} \cdot\left(h_{n+1, m} \mathbf{u}\right)
$$

the integral can be expressed as a flow through the boundary of the face, that is through its edges.

We give the expression of this sum of line integrals along the edges of $\mathcal{F}$ in section 4 .

\subsection{Orthogonal part}

The expression of the orthogonal part comes from specific properties of function $h_{n+1, m} . I_{n \perp}$ can be expressed as the flow of $\nabla h_{n+1, m}$ through the face $\mathcal{F}$

$$
I_{n \perp}=\left(\mathbf{e}_{\perp} \cdot \mathbf{n}\right) \iint_{\mathcal{F}} \nabla h_{n+1, m} \cdot \mathbf{d} \mathbf{S}
$$

Let $\{\mathcal{E}\}_{\mathcal{E} \in \partial \mathcal{F}}$ be the set of the edges of $\mathcal{F}$. For each edge $\mathcal{E}$, let us consider the triangular face $(O, \mathcal{E})$ defined by the origin $O$ of the frame and the edge itself, as shown in figure 3 . Let us consider the polyhedron $\mathcal{V}_{\mathcal{F}}$ defined by the set of faces $\left\{\mathcal{F},\{(O, \mathcal{E})\}_{\mathcal{E} \in \partial \mathcal{F}}\right\}$.

Since $h_{n+1, m}$ is harmonic it will hold

$$
\oiint_{\partial \mathcal{V}_{\mathcal{F}}} \nabla h_{n+1, m} \cdot \mathbf{d} \mathbf{S}=0
$$

where $\mathbf{d S}$ denote the vector elementary surface normal to the surface, and $\partial \mathcal{V}_{\mathcal{F}}$ is the whole surface of the volume $\mathcal{V}_{\mathcal{F}}$ associated to face $\mathcal{F}$

$$
\partial \mathcal{V}_{\mathcal{F}} \equiv\left\{\mathcal{F},\{(O, \mathcal{E})\}_{\mathcal{E} \in \partial \mathcal{F}}\right\}
$$

As a consequence

$$
\begin{aligned}
\iint_{\mathcal{F}} \nabla h_{n+1, m} \cdot \mathbf{d} \mathbf{S} & =-\sum_{\mathcal{E} \in \partial \mathcal{F}} \iint_{(0, \mathcal{E})} \nabla h_{n+1, m} \cdot \mathbf{d} \mathbf{S} \\
& =-\sum_{\mathcal{E} \in \partial \mathcal{F}} \iint_{(0, \mathcal{E})} \nabla h_{n+1, m} \cdot \mathbf{n}_{(0, \mathcal{E})} d S
\end{aligned}
$$




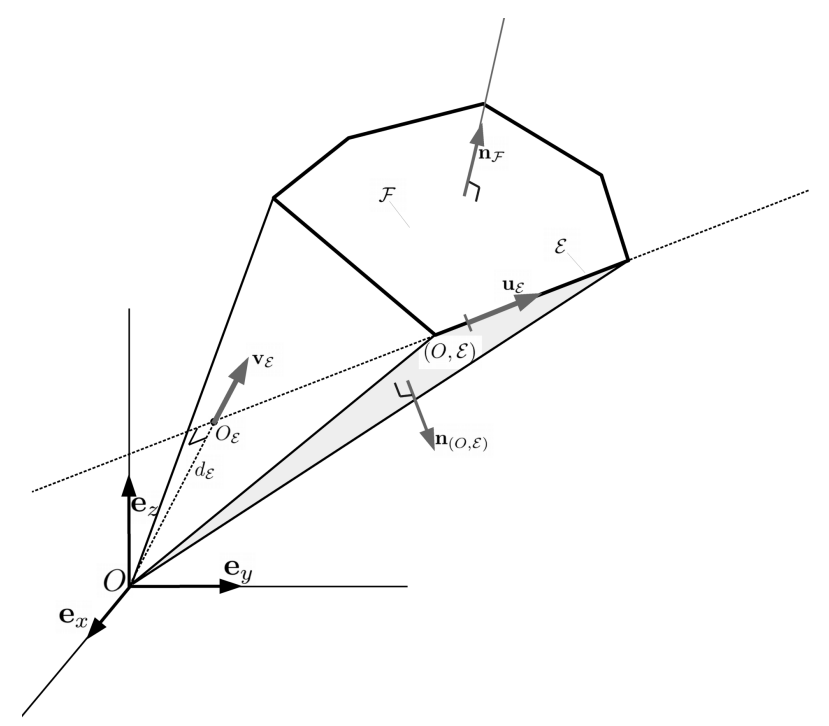

Figure 3: Volume $\mathcal{V}_{\mathcal{F}}$ associated to face $\mathcal{F}$

where $\mathbf{n}_{(0, \mathcal{E})}$ is the outgoing normal vector of the face $(0, \mathcal{E})$ (figure 3 ) and $\mathbf{d} \mathbf{S} \equiv d S \mathbf{n}_{(0, \mathcal{E})}$.

Let us consider $\nabla h_{n+1, m} \cdot \mathbf{n}$ as a function defined in the plane of the face $(O, \mathcal{E})$, expressed in cartesian coordinates as

$$
\nabla h_{n+1, m} \cdot \mathbf{n}_{(0, \mathcal{E})} \equiv f(u, v)
$$

$f(u, v)$ is an homogeneous function of degree $n$ which verifies the Euler identity

$$
n f(u, v)=u \frac{\partial f}{\partial u}+v \frac{\partial f}{\partial v}
$$

and thus the following relation for any point $P$ in face $(0, \mathcal{E})$

$$
(n+2) f(P)=\nabla_{2}(f(P) \mathbf{O P})
$$

As a consequence, each integral of the sum of the right member of equation 20 can be expression as the flow of $(f(P) \mathbf{O P})$ through the boundary of the face $(O, \mathcal{E})$, that is as a sum of line integrals. An simpler expression of this sum of line integrals is proposed in section 4 by expressing $\nabla h_{n+1, m}$ as the rotational of a vector field.

\section{Analytical formulation}

Vectors $\mathbf{e}_{\perp}$ and $\mathbf{e}_{/ /}$can be expressed as

$$
\begin{aligned}
\mathbf{e}_{\perp} & =\left(\mathbf{e}_{z} \cdot \mathbf{n}\right) \mathbf{n} \\
\mathbf{e}_{/ /} & =\left(\mathbf{n} \times \mathbf{e}_{z}\right) \times \mathbf{n}
\end{aligned}
$$




\subsection{Parrallel part}

Relation 25 leads to

$$
\begin{aligned}
I_{n / l} & =\iint_{\mathcal{F}} \nabla h_{n+1, m} \cdot\left(\left(\mathbf{n} \times \mathbf{e}_{z}\right) \times \mathbf{n}\right) d S \\
& =\iint_{\mathcal{F}}\left(\nabla h_{n+1, m} \times\left(\mathbf{n} \times \mathbf{e}_{z}\right)\right) \cdot \mathbf{n} d S
\end{aligned}
$$

Since the vector $\mathbf{n} \times \mathbf{e}_{z}$ is constant over the face $\mathcal{F}$, we can write

$$
\nabla h_{n+1, m} \times\left(\mathbf{n} \times \mathbf{e}_{z}\right)=\nabla \times\left(h_{n+1, m}\left(\mathbf{n} \times \mathbf{e}_{z}\right)\right)
$$

By applying of the Kelvin-Stokes theorem, we have

$$
\begin{aligned}
I_{n / l} & =\oint_{\partial \mathcal{F}}\left(h_{n+1, m}\left(\mathbf{n} \times \mathbf{e}_{z}\right)\right) \cdot \mathbf{d} \mathbf{L} \\
& =\sum_{\mathcal{E} \in \partial \mathcal{F}} \int_{\mathcal{E}} h_{n+1, m}\left(\mathbf{n} \times \mathbf{e}_{z}\right) \cdot \mathbf{u}_{\mathcal{E}} d L \\
& =\sum_{\mathcal{E} \in \partial \mathcal{F}}\left[\mathbf{n}, \mathbf{e}_{z}, \mathbf{u}_{\mathcal{E}}\right] \int_{\mathcal{E}} h_{n+1, m} d L
\end{aligned}
$$

where $\mathbf{d L} \equiv d L \mathbf{u}_{\mathcal{E}}$ is the elementary length vector along edge $\mathcal{E}$ and $\left[\mathbf{n}, \mathbf{e}_{z}, \mathbf{u}_{\mathcal{E}}\right]$ is the triple product between these three vectors, defined as the determinant of the matrix composed by their corresponding components.

\subsection{Orthogonal part}

The proposed expression of the orthogonal part results from the following property of $\nabla h_{n, m}$ (see Appendix, section A.2)

$$
(n+1) \nabla h_{n, m}=\nabla \times\left(\nabla \times\left(h_{n, m} \mathbf{r}\right)\right)
$$

where $\mathbf{r} \equiv \mathbf{O P}$ denotes the vector from the origin to any point $P$ of the face.

As a consequence, denoting elementary surface and line elements as in the previous sections and using relation 24 , we can write

$$
I_{n \perp}=\frac{\left(\mathbf{e}_{z} \cdot \mathbf{n}\right)}{n+2} \iint_{\mathcal{F}} \nabla \times\left(\nabla \times\left(h_{n+1, m} \mathbf{r}\right)\right) \cdot \mathbf{d} \mathbf{S}
$$

and, in application of the Kelvin-Stokes theorem

$$
\begin{aligned}
I_{n \perp} & =\frac{\left(\mathbf{e}_{z} \cdot \mathbf{n}\right)}{n+2} \oint_{\partial \mathcal{F}}\left(\nabla \times\left(h_{n+1, m} \mathbf{r}\right)\right) \cdot \mathbf{d} \mathbf{L} \\
& =\frac{\left(\mathbf{e}_{z} \cdot \mathbf{n}\right)}{n+2} \sum_{\mathcal{E} \in \partial \mathcal{F}} \int_{\mathcal{E}}\left(\nabla h_{n+1, m} \times \mathbf{r}\right) \cdot \mathbf{u}_{\mathcal{E}} d L \\
& =\frac{\left(\mathbf{e}_{z} \cdot \mathbf{n}\right)}{n+2} \sum_{\mathcal{E} \in \partial \mathcal{F}} \int_{\mathcal{E}} \nabla h_{n+1, m} \cdot\left(\mathbf{r} \times \mathbf{u}_{\mathcal{E}}\right) d L
\end{aligned}
$$


Let $O_{\mathcal{E}}$ be the nearest point from the origin $O$ of the line bearing the edge $\mathcal{E}$ (figure 3 ), $d_{\mathcal{E}}$ the distance from the origin $O$ to this line and $\mathbf{v}_{\mathcal{E}}$ the unit vector of direction $\overrightarrow{O O_{\mathcal{E}}}$

$$
\begin{aligned}
d_{\mathcal{E}} & \equiv\left\|\overrightarrow{O O_{\mathcal{E}}}\right\| \\
\mathbf{v}_{\mathcal{E}} & \equiv\left\{\begin{array}{c}
\frac{1}{d_{\mathcal{E}}} \overrightarrow{O O_{\mathcal{E}}} \text { if } d_{\mathcal{E}}>0 \\
\overrightarrow{0} \text { if } d_{\mathcal{E}}=0
\end{array}\right.
\end{aligned}
$$

The cross product $\left(\mathbf{r} \times \mathbf{u}_{\mathcal{E}}\right)$ is constant along the edge and can be expressed as

$$
\mathbf{v}_{\mathcal{E}} \times \mathbf{u}_{\mathcal{E}}=d_{\mathcal{E}}\left(\mathbf{v}_{\mathcal{E}} \times \mathbf{u}_{\mathcal{E}}\right)
$$

Thus the orthogonal part can be expressed as a weighted sum of line integrals of the derivative of $h_{n+1}$ along the constant (but edge dependant) direction $\mathbf{v}_{\mathcal{E}} \times \mathbf{u}_{\mathcal{E}}$

$$
I_{n \perp}=\frac{\left(\mathbf{e}_{z} \cdot \mathbf{n}\right)}{n+2} \sum_{\mathcal{E} \in \partial \mathcal{F}} d_{\mathcal{E}} \int_{\mathcal{E}} \nabla h_{n+1, m} \cdot\left(\mathbf{v}_{\mathcal{E}} \times \mathbf{u}_{\mathcal{E}}\right) d L
$$

\section{Numerical implementation}

A prototype software implementing the method based on the relations given in section 4 was written in the interpreted language Python (van Rossum and Drake, 2011).

Coordinates $(x, y, z)$ where normalized by the reference radius $a:\left(x^{\prime}, y^{\prime}, z^{\prime}\right) \equiv$ $(x / a, y / a, z / a)$. Legendre functions were normalized with the usual normalisation factor following Heiskanen and Moritz (1967, p. 31)

$$
N_{n, m}=\sqrt{\left(2-\delta_{0, m}\right)(2 n+1) \frac{(n-m) !}{(n+m) !}}
$$

All the used relations were adapted to the computation of the cormalized coefficients

$$
\left[\overline{C_{n, m}}\right]=\left[\begin{array}{c}
C_{n, m} / N_{n, m} \\
S_{n, m} / N_{n, m}
\end{array}\right]
$$

through the integration of

$$
\overline{h_{n, m}}\left(x^{\prime}, y^{\prime}, z^{\prime}\right)=\frac{N_{n, m}}{a^{n}} h_{n, m}(x, y, z)
$$

Line integrals were computed thought the decimation of edges of the test body into $2^{k}$ segments, the computation of $\overline{h_{n, m}}$ and its derivatives at the $2^{k}+1$ end points of the semgents, and the use of Romberg's integration method (Romberg, 1955) as implemented in the scipy.integrate package of Python (function $\operatorname{romb}())$. The exponent $k$ was chosen as a function of the maximum computed spherical harmonic degree $n_{\max }$, the lenght $l$ of the considered edge and 


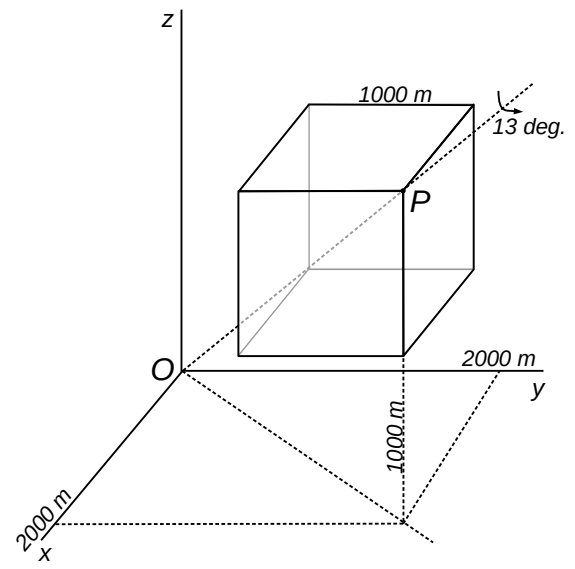

Figure 4: Test body

of the average distance $\bar{d}$ of its extremities to the orgin of the frame, such as

$$
\frac{l}{2^{k}} \leq \frac{1}{2} \frac{\pi}{n_{\max }} \bar{d}
$$

In this formula, $\pi \bar{d} / n_{\max }$ gives an raw approximate value of the resolution of the spherical harmonic model on the considered edge. The factor $1 / 2$ aims at dividing the edge beyond this resolution.

\section{Test body and reference values.}

We chose as test body a cube of size 1000 meters, centered on the point $(1500,1500,1500)$, and rotated by 13 degres around the diagonal passing through the origin (figure 4). The cube was located away from the center of the frame and rotated to avoid symetries that would cause many spherical harmonic coefficients to vanish. The density of the cube was arbitrarily set to $2.67 \mathrm{~g} \mathrm{~cm}^{-3}$.

The computed spherical harmonic series were compared to the value of the potential and of the gravity on a single point $P$ located at the furthest corner from the origin (see figure 4). The distance $O P$ was chosen as the reference radius $a$ for the computation of the spherical harmonic coefficients. The point $P$ was chosen at a corner in order to test a region of the space where high spatial frequencies are important.

The potential and gravitationnal attraction of the cube at point $P$ was derived from the exact formulas for the prism (Nagy et al., 2000), by expressing the limit of the expression given by Nagy et al. when the computation point reaches the corner $P$. The obtained reference values are

$$
V_{\text {cube }}(t)=G \rho\left(3 \log \left(\frac{1+\sqrt{3}}{\sqrt{2}}\right)-\frac{\pi}{4}\right) t^{2}
$$




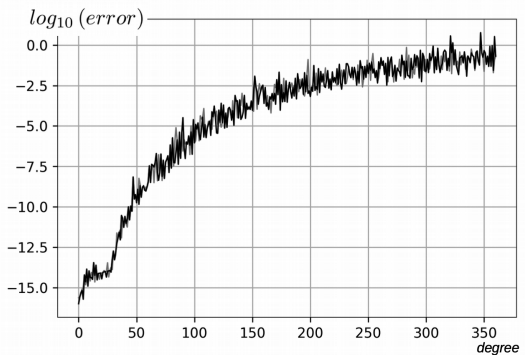

Figure 5: $\log _{10}$ of the relative error of the $\overline{h_{n, m}}$ line integrals on the vertical edges of the cube ; grey line: for $\overline{h_{n, m}^{c}}$; black line: for grey line : for $\overline{h_{n, m}^{s}}$

$$
g_{\text {cube }}(t)=G \rho\left(\frac{\pi}{6}+2 \log \left(\sqrt{2} \frac{1+\sqrt{2}}{1+\sqrt{3}}\right)\right) \sqrt{3} t
$$

where $t$ si the size of the cube, $G$ the gravitationnal constant, and $\rho$ the volumic mass of the cube. For our given example, we had $V_{\text {cube }}=2.12062410^{-1} \mathrm{~m}^{2} \mathrm{~s}^{-2}$ and $g_{\text {cube }}=2.99199610^{-4} \mathrm{~ms}^{-2}$.

\section{Results.}

The coefficient for our test cube were computed up to degree and order 360 . The strategy adopted for splitting the edges of the cube led to 129 computation points per edge. The overall computation of the spherical harmonic coefficients took one hour and a half on a computer with 8 Gbytes of RAM and a quad-core $2.5 \mathrm{GHz}$ Intel Core i7 processor.

We controlled the accuracy of the integrals on the vertical edges, for which an analytical solution is available through relation 7 .

Figure 5 shows the base 10 logarithm of the relative error of the integrals on vertical edges. The relative error is computed as the difference between the numerical value of the integrals and its reference value obtained from its analytical formula, divided by this reference value. The errors grow steadily up to nearly $100 \%$ at degree 360 . We note that the adopted numerical integration strategy looks sufficient up to degree 100 to 150 , but should be improved to properly handle computations at dergee and order 360 or more. Nevertheless, we considered it as sufficient to test the behavious of the method up to that degree.

Table 1 shows some of the computed coefficients. The values of $\overline{C_{1,0}}, \overline{C_{1,1}}$ and $\overline{S_{1,1}}$ are coherent with the position of the center of mass of the cube at the $10^{-14}$ level of relative accuracy.

The convegence of the spherical harmonic series toward the total values at test point $P$ (figure 4 ), given by the equations 39 and 40 , is shown on the figure 6 , for the potential and for the gravitationnal attraction. The evolution of the values toward the full potential and attraction is steady and does not seem to 


\begin{tabular}{|c|c|c|c|}
\hline$n$ & $m$ & $\overline{C_{n, m}}$ & $\overline{S_{n, m}}$ \\
\hline \hline 1 & 0 & $2.50000000000 e-01$ & 0.0 \\
\hline 1 & 1 & $2.50000000000 e-01$ & $2.50000000000 e-01$ \\
\hline 2 & 0 & $-6.28013820649 e-15$ & 0.0 \\
\hline 2 & 1 & $1.45236875483 e-01$ & $1.45236875483 e-01$ \\
\hline 2 & 2 & $3.86367158115 e-14$ & $1.45236875483 e-01$ \\
\hline$\ldots$ & $\ldots$ & $\ldots$ & $\ldots$ \\
\hline 180 & 0 & $-1.01265250801 e-08$ & 0.0 \\
\hline 180 & 2 & $-7.23646197302 e-12$ & $1.48621884351 e-08$ \\
\hline$\ldots$ & $\ldots$ & $\ldots$ & $\ldots$ \\
\hline 360 & 1 & $-2.52287113787 e-09$ & $-2.51541751845 e-09$ \\
\hline 360 & 360 & $-5.51466428191 e-41$ & $5.14086641792 e-40$ \\
\hline
\end{tabular}

Table 1: Spherical harmonic coefficients of the test cube (sample)

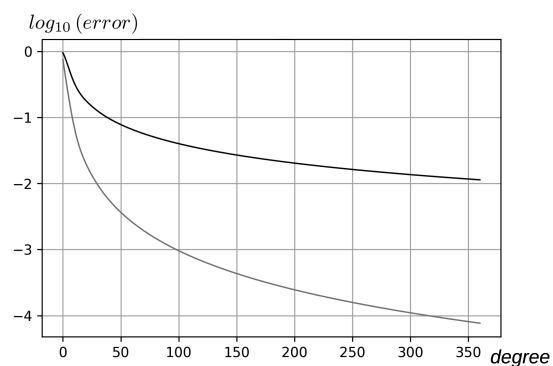

Figure 6: Base $10 \log$ of the diffence between the total reference value and the value computed from the expansion of the spherical harmonic serie up to a given degree as a function of the degree; grey line: potential; black line: gravitationnal attraction.

show any artifact. Of course, the relative error of the serie developed up to degree and order 360 is better for the potential value $\left(<10^{-4}\right)$ that for the acceleration $\left(>10^{-2}\right)$, since the derivation enhances high frequencies.

\section{Concluding remarks}

We presented here a new method for computing the spherical harmonic coefficients of a general polyhedron of constant density and tested it on a simple cubic shape. We gave original analytical expressions of theses coefficients as sums of line integrals. This finding voids the necessity for either handling complex and unstable recurrent computations as in (Tsoulis et al., 2009) or splitting the body and rotating its parts as in (Chen et al., 2019).

The complexity of the proposed method ranges between the algorithm by Werner (1997), the computation burden of which varies like $n^{4}$ to get all the 
coefficients at degree and order $n$, and the one by Tsoulis et al. (2009) or Chen et al. (2019) the computation buden of which varies like $n^{2}$. In our case, the number of points computed to sample the edges of the body is of course a function of the aimed resolution, and thus of the wished maximum degree. This make the method in the order of complexity of $n^{3}$. The processing time mentionned in the section 5 was given as an indication that the computation is possible. It has though no significant meaning. First, our prototype computation was handled though an interpreted langage. And secondly, the method can of course be highly parallelized.

The tests presented in section 5show that our method allows to compute the spherical harmonic coefficients of a simple body at least up to degree and order 360 with a better stability than previous methods. The method proposed by Tsoulis et al. (2009) failed to provide accurate coefficients beyond degree 60 . It was though assessed on a much more complex body. The tests presented by Chen et al. (2019) on a prism with variable density did not allow to assess the behaviour of their method up to the same degree as here. Chen et al. chose the computation point at a distance of $1.1078 a$, $a$ being the reference radius, which minimize the effect of very high frequencies due to the factor $(1 / 1.1078)^{n}$. It yields a convergence to the reference value as soon as degree 150 for the potential, 240 for the acceleration and 290 for the second derivatives, which prevents from assessing the quality of higher coefficients. Besides, the convergence cuves shown in their work appear very noisy compared to the one we observe with our method.

This good behaviour of the convergence curves (figure 6) lets us expect a good accuracy of the coefficients. Besides, the methods can still be improved, since our tests show also that the currently adopted line integration strategy presents some limits at high degrees.

Further works will focus on deeper numerical experiments in order to assess the accuracy of the computed coefficients, as well as to study the behaviour of the method on more realistic bodies.

Acknowledgements This study was partly supported by IdEx Université de Paris ANR-18-IDEX-0001. This is IPGP contribution number 4055.

\section{References}

Balmino, G. (1994), 'Gravitational potential harmonics from the shape of an homogeneous body', Celestial Mechanics and Dynamical Astronomy 60(3), 331364 .

Chao, B. F. and Rubincam, D. P. (1989), 'The gravitational field of phobos', Geophysical Research Letters 16(8), 859-862.

Chen, C., Chen, Y. and Bian, S. (2019), 'Evaluation of the spherical harmonic coefficients for the external potential of a polyhedral body with linearly varying density', Celestial Mechanics and Dynamical Astronomy 131(2), 8. 
De Loera, J. A., Dutra, B., Köppe, M., Moreinis, S., Pinto, G. and Wu, J. (2013), 'Software for exact integration of polynomials over polyhedra', Computational Geometry 46, 232-252.

Heiskanen, W. A. and Moritz, H. (1967), Physical Geodesy, WH Freeman and Company, San Fransisco.

Jamet, O. and Thomas, E. (2004), A linear algorithm for computing the spherical harmonic coefficients of the gravitational potential from a constant density polyhedron, in CNES, ed., 'Proceedings of the $2^{\text {nd }}$ International GOCE User Workshop, 'GOCE, the geoid and oceanography".

Lasserre, J. B. (1999), 'Integration and homogeneous functions', Proceedings of the American Mathematical Society 127(3), 813-818.

Martinec, Z., Peck, K. and Bursa, M. (1989), 'The phobos gravitational field modeled on the basis of its topography.', Earth Moon Planets 45, 219-235.

Nagy, D., Papp, G. and Benedek, J. (2000), 'The gravitational potential and its derivatives for the prism', Journal of Geodesy 74(7-8), 552-560.

Romberg, W. (1955), 'Vereinfachte numerische integration', Det Kongelige Norske Videnskabers Selskab Forhandlinger 28(7), 30-36. Trondheim.

Tsoulis, D., Jamet, O., Verdun, J. and Gonindard, N. (2009), 'Recursive algorithms for the computation of the potential harmonic coefficients of a constant density polyhedron', Journal of Geodesy 83(10), 925-942.

van Rossum, G. and Drake, F. L. (2011), The Python Language Reference Manual, Network Theory Ltd.

Werner, R. A. (1997), 'Spherical harmonic coefficients for the potential of a constant-density polyhedron', Computers \& Geosciences 23(10), 1071-1077.

\section{Appendix}

\section{A.1 Derivative of $h_{n, m}$ along the $z$ axis}

The relation expressed by equation 7 is obtained in polar coordinates as follows (the calculus is identical with a $\sin (m \lambda)$ dependency to the longitude)

$$
\begin{aligned}
\frac{\partial h_{n, m}^{c}(\rho, \lambda, z)}{\partial z}= & \frac{\partial}{\partial z}\left({\sqrt{\rho^{2}+z^{2}}}^{n} P_{n, m}\left(\frac{z}{\sqrt{\rho^{2}+z^{2}}}\right) \cos (m \lambda)\right) \\
= & \frac{\partial{\sqrt{\rho^{2}+z^{2}}}^{n}}{\partial z} P_{n, m}\left(\frac{z}{\sqrt{\rho^{2}+z^{2}}}\right) \cos (m \lambda) \\
& +{\sqrt{\rho^{2}+z^{2}}}^{n} \frac{\partial}{\partial z} P_{n, m}\left(\frac{z}{\sqrt{\rho^{2}+z^{2}}}\right) \cos (m \lambda)
\end{aligned}
$$


We have

$$
\frac{\partial{\sqrt{\rho^{2}+z^{2}}}^{n}}{\partial z}=n z{\sqrt{\rho^{2}+z^{2}}}^{(n-2)}
$$

and

$$
\begin{gathered}
\frac{\partial}{\partial z} P_{n, m}\left(\frac{z}{\sqrt{\rho^{2}+z^{2}}}\right)=\frac{1}{\sqrt{\rho^{2}+z^{2}}}\left(1-\left(\frac{z}{\sqrt{\rho^{2}+z^{2}}}\right)^{2}\right) . \\
P_{n, m}^{\prime}\left(\frac{z}{\sqrt{\rho^{2}+z^{2}}}\right)
\end{gathered}
$$

where $P_{n, m}^{\prime}$ is the first derivative of the polynomial $P_{n, m}: P_{n, m}^{\prime}(x)=\frac{d}{d x} P_{n, m}(x)$.

Using the relation

$$
\left(x^{2}-1\right) \frac{d}{d x} P_{n, m}(x)=n x P_{n, m}(x)-(n+m) P_{n-1, m}(x)
$$

we can write

$$
\begin{aligned}
\frac{\partial}{\partial z} P_{n, m}\left(\frac{z}{\sqrt{\rho^{2}+z^{2}}}\right)= & \frac{n+m}{\sqrt{\rho^{2}+z^{2}}} P_{n-1, m}\left(\frac{z}{\sqrt{\rho^{2}+z^{2}}}\right) \\
& -n \frac{z}{\sqrt{\rho^{2}+z^{2}}} P_{n, m}\left(\frac{z}{\sqrt{\rho^{2}+z^{2}}}\right)
\end{aligned}
$$

and consequently

$$
\frac{\partial h_{n, m}^{c}(\rho, \lambda, z)}{\partial z}=(n+m){\sqrt{\rho^{2}+z^{2}}}^{(n-1)} P_{n-1, m}\left(\frac{z}{\sqrt{\rho^{2}+z^{2}}}\right) \cos (m \lambda)
$$

\section{A.2 Gradient of $h_{n, m}$ as a rotationnal}

Starting from the identity

$$
\nabla \times(\nabla \times \mathbf{F})=\nabla(\nabla . \mathbf{F})-\triangle \mathbf{F}
$$

we have

$$
\nabla \times\left(\nabla \times\left(h_{n, m} \mathbf{r}\right)\right)=\nabla\left(\nabla \cdot\left(h_{n, m} \mathbf{r}\right)\right)-\Delta\left(h_{n, m} \mathbf{r}\right)
$$

From the homogeneity of $h_{n, m}$, we get, through the Euler relation

$$
\nabla \cdot\left(h_{n, m} \mathbf{r}\right)=(n+3) h_{n, m}
$$

and thus

$$
\nabla\left(\nabla \cdot\left(h_{n, m} \mathbf{r}\right)\right)=(n+3) \nabla h_{n, m}
$$


The second term of the relation 48 can be written in cartesian coordinates

$$
\begin{aligned}
\Delta\left(h_{n, m} \mathbf{r}\right)= & \left(\begin{array}{c}
\triangle\left(x h_{n, m}\right) \\
\triangle\left(y h_{n, m}\right) \\
\triangle\left(z h_{n, m}\right)
\end{array}\right) \\
= & \left(\begin{array}{c}
2 \nabla h_{n, m} \cdot \mathbf{e}_{x}+x \triangle h_{n, m} \\
2 \nabla h_{n, m} \cdot \mathbf{e}_{y}+y \triangle h_{n, m} \\
2 \nabla h_{n, m} \cdot \mathbf{e}_{z}+z \triangle h_{n, m}
\end{array}\right) \\
= & 2 \nabla h_{n, m}+\left(\triangle h_{n, m}\right) \mathbf{r}
\end{aligned}
$$

Since $h_{n, m}$ is harmonic, $\triangle h_{n, m}=0$ and we get

$$
\nabla \times\left(\nabla \times\left(h_{n, m} \mathbf{r}\right)\right)=(n+1) \nabla h_{n, m}
$$

